\title{
Mental rotation versus invariant features in object perception from different viewpoints: an fMRI study
}

\author{
Jan Vanrie $^{\mathrm{a}}$, Erik Béatse ${ }^{\mathrm{b}}$, Johan Wagemans ${ }^{\mathrm{a}, *}$, Stefan Sunaert ${ }^{\mathrm{b}}$, Paul Van Hecke ${ }^{\mathrm{b}}$ \\ a Department of Psychology, University of Leuven, Tiensestraat 102, B-3000 Leuven, Belgium \\ ${ }^{\mathrm{b}}$ Department of Radiology, University Hospital Gasthuisberg, University of Leuven, Leuven, Belgium
}

Received 8 January 2001; accepted 25 July 2001

\begin{abstract}
It has been proposed that object perception can proceed through different routes, which can be situated on a continuum ranging from complete viewpoint-dependency to complete viewpoint-independency, depending on the objects and the task at hand. Although these different routes have been extensively demonstrated on the behavioral level, the corresponding distinction in the underlying neural substrate has not received the same attention. Our goal was to disentangle, on the behavioral and the neurofunctional level, a process associated with extreme viewpoint-dependency, i.e. mental rotation, and a process associated with extreme viewpoint-independency, i.e. the use of viewpoint-invariant, diagnostic features. Two sets of 3-D block figures were created that either differed in handedness (original versus mirrored) or in the angles joining the block components (orthogonal versus skewed). Behavioral measures on a same-different judgment task were predicted to be dependent on viewpoint in the rotation condition (same versus mirrored), but not in the invariance condition (same angles versus different angles). Six subjects participated in an fMRI experiment while presented with both conditions in alternating blocks. Both reaction times and accuracy confirmed the predicted dissociation between the two conditions. Neurofunctional results indicate that all cortical areas activated in the invariance condition were also activated in the rotation condition. Parietal areas were more activated than occipito-temporal areas in the rotation condition, while this pattern was reversed in the invariance condition. Furthermore, some areas were activated uniquely by the rotation condition, probably reflecting the additional processes apparent in the behavioral response patterns. (C) 2002 Elsevier Science Ltd. All rights reserved.
\end{abstract}

Keywords: Object recognition; Orientation specificity; Neuroimaging

\section{Introduction}

An intriguing feature of our visual system is the efficiency with which it is able to recognize objects, even when seen from different viewpoints. The issue of object recognition has been investigated extensively and several theoretical models have been proposed to account for this capacity. Over the years, the theoretical accounts as well as the way in which they are tested have evolved significantly. However, if one reviews the recent literature on the topic, two trends are especially prevalent.

The first trend can be described as a shift in the focus of discussion. In previous years, the debate was primarily concentrated on whether object recognition was "viewpoint-dependent" (e.g. [43]) or "viewpoint-independent" (e.g. [4]). In this debate, the two "sides" have used a variety of stimuli, experimental paradigms and techniques to test the predictions generated by their models and both, in

* Corresponding author. Tel.: +32-16-325969; fax: +32-16-326099.

E-mail address: johan.wagemans@psy.kuleuven.ac.be (J. Wagemans). fact, provided substantial empirical evidence in their favor. Given this growing amount of conflicting results, it became increasingly difficult to hold the view of a single, unitary system, which operates in a fixed manner. For this reason, notions such as "multiple routes to object recognition" are adopted more and more frequently in vision science in recent years (e.g. [28]). More specifically, there seems to be a growing consensus that there is a continuum from viewpoint-independency to viewpoint-dependency, which is in part influenced by stimulus discriminability and the task at hand (e.g. $[3,12,19,20,47])$. In short, the key question is no longer if object recognition is viewpoint-dependent or viewpoint-independent, but rather when, i.e. under which circumstances.

The second important trend is more general and consists of the increasing attention for the neurofunctional correlates of mental processes, including processes underlying visual perception. This line of research has obviously been strongly stimulated by the advent of neuroimaging techniques like PET and fMRI (for reviews see $[10,15,44,54])$. 
If we take these two trends into account, it is somewhat surprising that relatively little research has been done on the cortical areas associated with different routes to object recognition. This is precisely the main goal of the present investigation. Although some neuroimaging studies have addressed the issue of perceiving objects from different viewpoints (e.g. [18,26,38]), there have been-to our knowledge-no studies that explicitly tried to contrast these routes on the neurofunctional level. ${ }^{1}$ Nevertheless, differences in the underlying cortical activations would provide strong convergent evidence that there are indeed distinct processes involved.

In order to investigate neurofunctional correlates of different routes to object perception, we first need to create circumstances in which different processes will be invoked.

Fortunately, we know of such circumstances in which recognition is undoubtedly viewpoint-dependent or viewpoint-independent. An extreme example of the former is the case of mental rotation, while the latter is clearly obtained when the visual system makes use of locally diagnostic, viewpoint-invariant features.

\subsection{Mental rotation}

A special case in object recognition is the situation in which the visual system needs to distinguish an object from its mirrored counterpart. It has been shown that in this case, the visual system engages in a process of mental rotation. This visuo-spatial process, first described by Shepard and Metzler [37], yields very robust viewpoint-dependent effects: ${ }^{2}$ response times in a same-different judgment increased linearly with increasing angular difference (AD) between two comparison objects. Although making such a distinction might not be a typical object recognition task (see also $[42,58]$ ), it nevertheless, "involves extensive recognition and comparison of objects and object parts" ([5], p. 11, on the Shepard and Metzler paradigm). ${ }^{3}$ Because a detailed computational model of the mental rotation paradigm is beyond the scope of the present study (see [5,22]) we will focus on one aspect relevant to the issue at hand: the use of a viewer-centered reference frame. Obviously, advo-

\footnotetext{
${ }^{1}$ To illustrate this point, while Vecera [49] has recently reviewed the literature on object representation and invariances in the context of a special issue on neuroimaging, he mentioned only Kosslyn et al. [26] as a neuroimaging study directly relevant to the viewpoint- versus object-centered debate.

${ }^{2}$ Although the coupling of viewpoint-dependency and increased response times on the one hand, and viewpoint-independency and no effect on response times on the other hand is widely accepted, some evidence suggests that this assumption should be treated with caution [52].

3 It is worth stressing that we do not assume that our "mental rotation" condition recruits all forms of viewpoint-dependent process; nor do we assert that our "invariance" condition taps into all viewpoint-independent recognition processes. What we do claim, however, is that both conditions encompass a number of processes relevant to the issue of perceiving objects from different perspectives, and that the two conditions are representative of viewpoint-dependent and viewpoint-independent routes to object recognition, as distinguished in the literature.
}

cates of viewpoint-dependent theories are likely to endorse the use of such a reference frame. Since handedness is not encoded in object-centered representations $[4,40]$, the use of a viewer-centered frame of reference becomes necessary in case of handedness discrimination, even according to viewpoint-independent theories. More specifically, the computation of the spatial coordinates of the object being mentally rotated will proceed from the specific viewpoint in which the observer perceives the objects. This makes the mental rotation process a highly appropriate research subject in the present discussion.

\subsection{Use of invariant features}

If the mental rotation process is an extreme case of viewpoint-dependency, the process in which observers make use of locally diagnostic, view-invariant features to determine the identity of an object (e.g. [13]), can be seen as an equally extreme case of viewpoint-independent object processing. Again, this process is not the most typical for everyday object recognition, but the operation of detecting and exploiting image invariances is clearly an important and relevant topic (e.g. [53]). Moreover, in the present experiment, top-down influences are likely to come into play, because the observers know what kind of feature is crucial to solve the task. That is, it is advantageous for task performance to direct attention to those locations in which the feature is present. In this sense, making use of diagnostic viewpoint-invariant features cannot be reduced to simple shape discrimination.

By including these rather extreme processes, mental rotation and the use of invariant, diagnostic features, we can expect clear and unambiguous behavioral results. In addition, neuropsychological investigations also demonstrated that the two processes can be selectively impaired. This constitutes a strong indication that-at least in brain damaged patients-they are indeed associated with different neural systems. For example, patient R.T., described by Farah and Hammond [16], was still able to recognize misoriented objects, despite his inability to perform mental rotation (see also $[8,11,24])$. Turnbull and McCarthy [46], on the other hand, reported the opposite pattern of a patient who succeeded on mental rotation tasks, but failed to recognize disoriented objects. Moreover, the double dissociation between "recognition by axes" and "recognition by features" (as described by Humphreys and Riddoch [21]) demonstrates the functional independence of a recognition process based on the detection and use of a distinctive feature when subjects are matching objects from different viewpoints. Four of the patients in the study by Humphreys and Riddoch [21] were able to match objects that were rotated in the plane even though the saliency of their primary distinctive feature was reduced. On the other hand, when shown the objects with their principal axis foreshortened, leaving the visibility of the distinctive feature unaltered, they failed to make the match. The reverse pattern was found in a fifth patient. 
Now, although these neuropsychological data strongly suggest that the two processes and their representations are also differentially distributed in the brain, the issue is not fully resolved. First of all, the relevant neuropsychological studies do not provide much neuroanatomical details regarding the lesion sites, so it is virtually impossible to derive more general insight into the specific cerebral areas involved in these processes. Furthermore, the idea of multiple routes could be strengthened considerably if supported by similar evidence in healthy participants. Although several studies reported evidence for the existence of different routes to object recognition on the behavioral level, only in very few cases, was this dissociation apparent in the same study, in which a similar paradigm and similar stimuli were used to test the different routes. Given the strong effect on recognition of both the nature of the stimuli and the experimental task, controlling for these two variables becomes crucial. Our main goal was, thus, to distinguish, both on the behavioral and on the neurofunctional level, two different routes to object perception in a single study in which the nature of the stimuli and the paradigm are equated as much as possible.

On the basis of previous studies on mental rotation (e.g. $[5,7,36,39])$, we can expect the major focus to be situated in the parietal area (Brodmann area (BA) 7), along with activity in frontal areas (pre-central gyrus, posterior middle frontal gyrus, and supplementary motor area). For the invariance condition, on the other hand, the main point of activation is predicted to be in the occipito-temporal and temporal cortical areas (see [1,2,27,34,50,51]).

\section{Method}

\subsection{Subjects, stimuli and task}

Six right-handed, healthy volunteers participated in the study (three male, three female) ranging from 22 to 35 years (mean age was 25.2 years).

Subjects were presented with two experimental conditions, a mental rotation condition and a condition in which viewpoint-invariant features were used, which will be referred to as rotation and invariance condition, respectively. The experimental paradigm was identical in both, namely to decide whether two simultaneously presented stimuli were views of the same 3-D object irrespective of possible rotations in depth.

All nine objects in the standard set were front/back and left/right asymmetrical, solid objects, consisting of three small limbs that were attached face-to-face onto a main limb (see, e.g. Fig. 1). In addition, we constructed a specific set of objects for each condition. For the rotation condition, we created enantiomorphic versions of the standard objects (further on referred to as "mirror" objects) by relocating two side-limbs to the opposite side of the main limb. For the invariance condition, the angle of attachment between the side-limbs and the main limb was altered, so that the
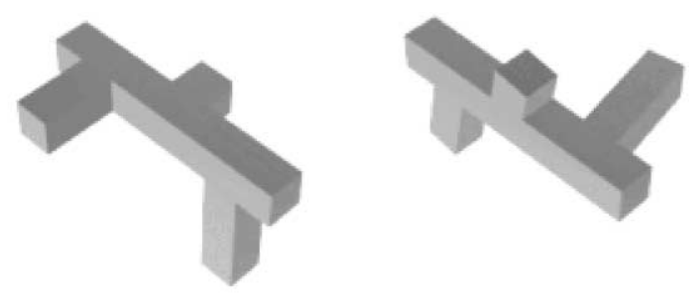

(a)
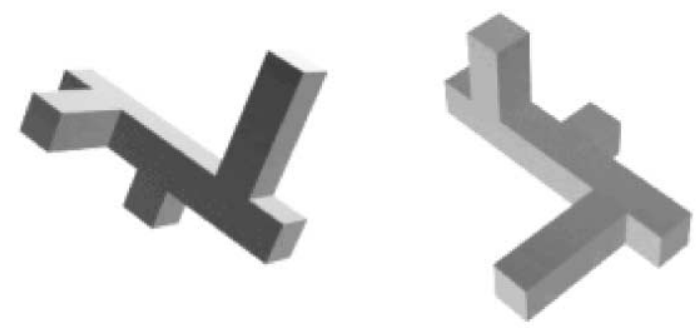

(b)

Fig. 1. Examples of non-match stimuli (a) in the invariance condition, with an $\mathrm{AD}$ of $-90^{\circ}$ (left object: standard $0^{\circ}$, right object: skewed $270^{\circ}$ ) and (b) in the rotation condition, with an $\mathrm{AD}$ of $+60^{\circ}$ (left object: standard $120^{\circ}$, right object: mirrored $180^{\circ}$ ).

attachment of a smaller limb was now no longer orthogonal (see Fig. 1a, right object). The side-components were tilted upward or downward by $10^{\circ}$. This angle was based on the results of a pilot study, set up to find the most appropriate amount of skewing, in which we observed that decreasing the amount of skewing increased mean response time, but reduced accuracy at the same time. The equilibrium in the trade-off between speed and accuracy of performance was situated exactly on the $10^{\circ}$ level of skewing angle. The relatively small, but qualitative difference was, thus, the angle formed by a small limb and the main limb: this was orthogonal $\left(90^{\circ}\right)$ in one set of objects and skewed $\left(80^{\circ} / 100^{\circ}\right)$ in the other set of objects.

We produced 12 different images of each of the nine standard, nine "mirrored", and nine "skewed" objects by rotating each object around its length-axis in steps of $30^{\circ}$. The images were copper-colored against a black background and subtended approximately $3.30^{\circ} \times 3.10^{\circ}$ of visual angle. Two views were shown simultaneously, next to one another, separated by $3.80^{\circ}$ of visual angle. All stimuli were presented on a partly translucent screen by way of a video projector.

\subsection{Procedure}

In both experimental conditions a stimulus pair was presented and remained visible until the subjects responded. Subjects judged whether the two views could stem from the same object or not. In the rotation condition, a non-match trial consisted of a view of an object and a view of its mirrored counterpart (Fig. 1b). In the invariance condition, the 
two objects were either both orthogonal or both skewed in case of a match trial, whereas for a non-match trial they differed in the angle of attachment of their side-components, i.e. one was orthogonal and the other skewed (Fig. 1a). Both speed and accuracy of the response were registered. The response was followed by a $500 \mathrm{~ms}$ black screen, after which a new stimulus pair appeared. Maximal presentation time was $7 \mathrm{~s}$, after which the stimuli disappeared automatically. Subjects responded by pushing one of the two response buttons. The hand used for pushing the 'same' button was counterbalanced across subjects.

The identity of the left object of a stimulus pair was determined in a pseudo-random order: each of the 18 different objects (nine standard and nine condition-specific) had to appear once before any other object could appear a second time, all had to appear twice before any other could appear a third time and so on (see [7] for a similar procedure). The same logic was used for the angular difference between the left and right view: each of the 12 possible angular differences $\left(30,60,90,120,150\right.$ and $180^{\circ}$ in both positive (clockwise) and negative (counterclockwise) direction) had to appear once, before a certain angular difference could appear a second time. In this way, we achieved an approximately equal frequency of all objects, and more importantly, all angular differences. Per set of 18 trials (in which 18 different left objects were presented), half consisted of matches, the other half of non-matches, with the constraint that no more than four consecutive trials could require the same response. Note that there were no trials in which both objects appeared at the same viewpoint $\left(0^{\circ}\right.$ of angular difference).

\subsection{Control condition}

The choice for an adequate control condition was somewhat restricted because the same control condition was to be used as a baseline for both experimental conditions. For example, this automatically excluded the 'traditional' control condition for the mental rotation task, in which the stimuli are presented at $0^{\circ}$ of angular difference. For this reason, we opted for a same-different judgment task using 2-D T-shaped stimuli. Subjects were presented with two T-figures, side-by-side on the screen, which could both be in the same orientation (match trial) or rotated $180^{\circ}$ from each other (non-match trial). For the T-figures, only four orientations were possible: an upright orientation $\left(0^{\circ}\right)$, inverted $\left(180^{\circ}\right)$, and two horizontal orientations $\left(90\right.$ and $\left.270^{\circ}\right)$. Half of the trials consisted of match trials $(0-0,90-90,180-180$, or $\left.270^{\circ}-270^{\circ}\right)$, the other half were non-match trials $(0-180$, $90^{\circ}-270^{\circ}$ ).

The reason why the objects were 2-D and did not differ by less than $180^{\circ}$ was to minimize the possibility that subjects would pay specific attention to the 3-D angles in the stimuli or inadvertently engage in a mental rotation process. Nonetheless, the "irrelevant" processes of encoding visual stimuli, deciding and executing a motor response were clearly present in this task.

\subsection{Design}

The three conditions (rotation, invariance and control) were presented in four types of time series with a 7-min duration. The first two time series consisted of twelve blocks of $35 \mathrm{~s}$ in which the control condition was alternated with either the rotation condition (CR) or the invariance condition (CI). In the other two time series, there was an alternation between the two experimental conditions. One series started with the rotation condition (RI), the other with the invariance condition (IR). Because the stimuli in these time series were so similar, subjects had to be informed when to start using a different response criterion, that is, when a new block started. Therefore, prior to each block subjects were presented with a 7-s indication image showing a view of a condition-specific object with an indication word ("mirror" or "skewed"). The sequence 'indication image 1-experimental condition 1-indication image 2-experimental condition 2' was then repeated five times.

In order to familiarize subjects with the stimuli, the task and the specific procedure of the experiment, all subjects performed each of the four possible time series $(\mathrm{CR}$, CI, RI, IR) once before entering the scanner. During the functional image acquisition, they performed each series twice, so in total, 12 sessions were administered. For half of the subjects, the ordering of the first four series was as follows: CR-CI-RI-IR. The ordering of series performed inside the scanner was then: CR-CI-CR-CI-RI-IR-RI-IR. For the other half of the subjects, the ordering was switched in pairs: CI-CR-IR-RI (outside the scanner) and CI-CR-CI-CR-IR-RI-IR-RI (inside the scanner).

\subsection{Imaging acquisition}

Imaging was performed using a Siemens Vision $1.5 \mathrm{~T}$ scanner and a standard quadrature headcoil. Anatomical images were acquired with a $T_{1}$-weighted MPRAGE sequence (using a $256 \times 256 \times 128$ matrix resulting in voxels with dimensions $1 \mathrm{~mm} \times 1 \mathrm{~mm} \times 1.250 \mathrm{~mm}$ resolution). A functional time series consisted of 125 scans of an echo-planar gradient-echo sequence $(\mathrm{TR}=3500 \mathrm{~ms}, \mathrm{TE}=40 \mathrm{~ms}$, FOV $=200 \mathrm{~mm}, 64 \times 64$ matrix, 32 interleaved transverse slices with slice thickness $4 \mathrm{~mm}$ and gap $0.5 \mathrm{~mm}$ ). The first five scans were not included in the dataset. The first stimulus was presented after the fifth scan. In one experimental session, eight functional time series were recorded. In total, subjects were in the scanner for approximately $2 \mathrm{~h}$.

\subsection{Analysis}

The analysis was performed on the data obtained while the subjects were inside the scanner. Prior to the analysis, $2.2 \%$ of the behavioral data were discarded as outliers. The behavioral data (both reaction times and accuracy) were then analyzed using a three-way repeated measures ANOVA 

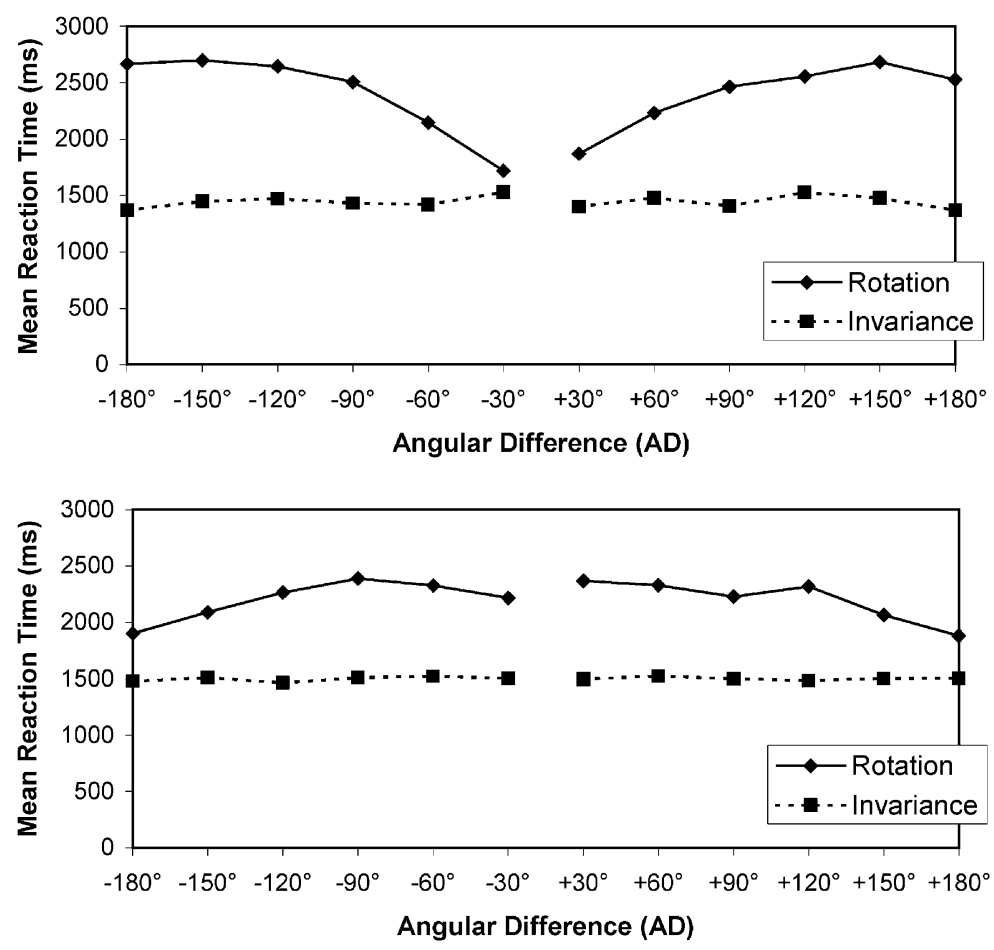

Fig. 2. Mean reaction time per angular difference (AD) on match trials (upper) and non-match trials (lower) for the two conditions.

with 'angular difference' (12 levels: $\pm 30, \pm 60, \ldots, \pm 180^{\circ}$ ), 'match' and 'process' (rotation and invariance) as independent variables. For reaction times, the analysis was performed on correctly responded trials only.
All analyses of imaging data were performed using SPM96 software (Functional Imaging Laboratory, Queen Square, London, UK). This included correction for head motion, spatial normalization to the Montreal Neurological
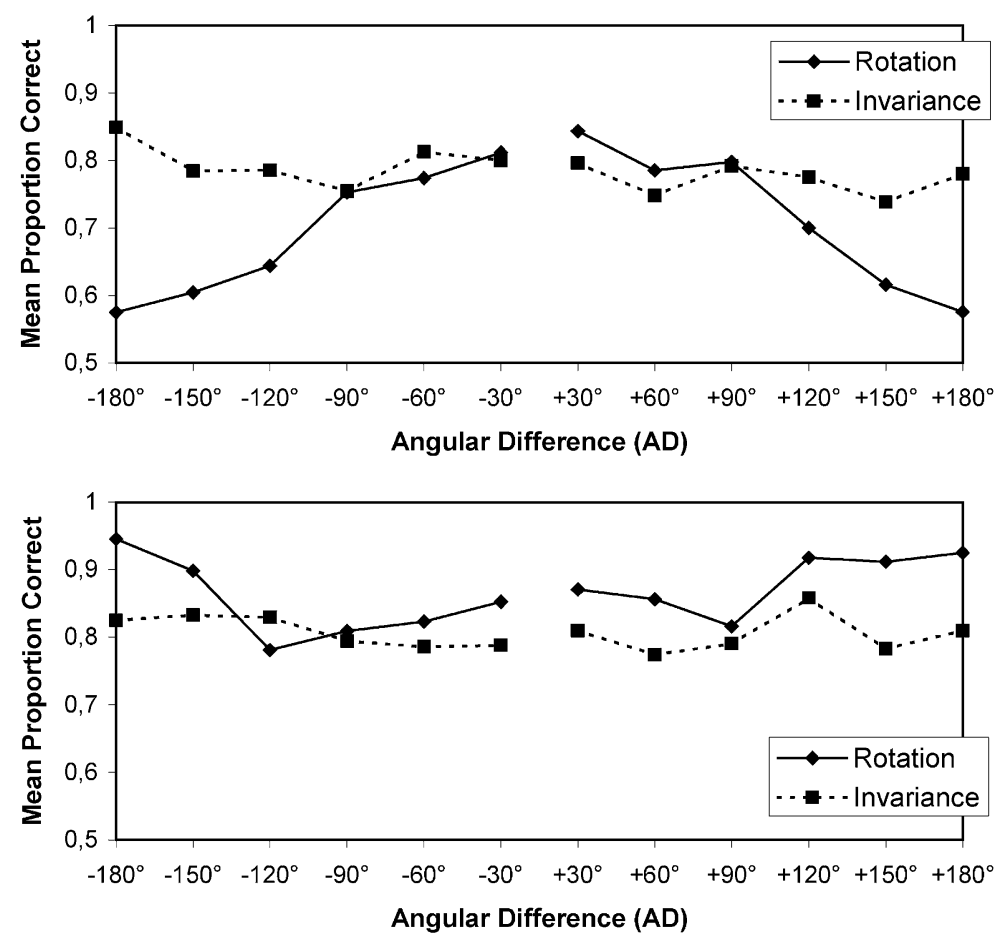

Fig. 3. Proportion correct responses per angular difference (AD) on match trials (upper) and non-match trials (lower) for the two conditions. 
Table 1

Talairach coordinates of the significantly activated areas for the group analysis and for individual subjects (S.) for four contrasts (I: invariance, R: rotation, C: control) $)^{\mathrm{a}}$

\begin{tabular}{|c|c|c|c|c|}
\hline S. & $\mathrm{I}-\mathrm{C}$ & $\mathrm{R}-\mathrm{C}$ & $\mathrm{I}-\mathrm{R}$ & R-I \\
\hline Group & $\begin{array}{l}\left({ }^{* 12},-\mathbf{7 0} 6\right) \mathbf{V 1} \\
(33,-87,-9) \text { iOG } \\
(* 36,-67,-22) \text { FG } \\
\left({ }^{* 28}, \mathbf{5 8}, \mathbf{5 5}\right) \mathbf{s P L} / \mathrm{IPS} \\
(\mathbf{3 0}, \mathbf{0}, \mathbf{5 1}) \mathbf{B A 6} \\
(\mathbf{3}, \mathbf{2 7}, \mathbf{4 5}) \mathbf{B A 8 m} \\
{[8.39]-[5.21]}\end{array}$ & $\begin{array}{l}(* 27, \mathbf{1}, \mathbf{5 4}) \mathbf{B A 6} \\
(51,39,27) \mathrm{BA} 9 / 46 \\
(* \mathbf{2 5},-\mathbf{5 7}, \mathbf{5 7}) \mathbf{s P L} \\
(* 37,-67,-24) \mathrm{FG} \\
(* 13,-60,4) \mathrm{V} 1 \\
(* 39,-36,39) \mathrm{iP} \\
(* 34,-78,16) \mathrm{iIPS} \\
(\mathbf{3}, \mathbf{3 0}, \mathbf{4 5}) \mathbf{B A 8 m} \\
{[8.75]-[6.86]}\end{array}$ & $\begin{array}{l}(-66,-6,-12) \text { BA21 } \\
(3,0,66) \text { BA6m } \\
(36,-21,66) \text { CS } \\
(60,9,-3) \text { BA44 } \\
(-69,-36,0) \text { BA22 } \\
{[6.61]-[4.89]}\end{array}$ & $\begin{array}{l}(\mathbf{2 1},-\mathbf{5 8}, \mathbf{6 0}) \mathbf{s P L} \\
(* 45,42,54) \mathrm{iP} / \mathrm{PoS} \\
(42,-72,21) \text { iIPS } \\
(\mathbf{2 8}, \mathbf{4}, \mathbf{6 1}) \mathbf{B A 6} \\
(-\mathbf{5 4},-\mathbf{6 0},-\mathbf{6}) \mathbf{V 5} \\
(\mathbf{3 3}, \mathbf{4 5},-\mathbf{9}) \mathbf{B A 4 7} \\
{[8.53]-[4.62]}\end{array}$ \\
\hline J.P. & $\begin{array}{l}(* \mathbf{4 2},-\mathbf{6 6},-\mathbf{2 3}) \mathbf{F G} \\
(* 31,-92,-2) \text { iOG } \\
(6,-86,-4) \text { V1 } \\
(\mathbf{4}, \mathbf{3 6}, \mathbf{4 6}) \mathbf{B A 8 m} \\
(\mathbf{4 8}, \mathbf{5 8}, \mathbf{2}) \mathbf{B A 1 0} \\
{[8.18]-[7.04]}\end{array}$ & 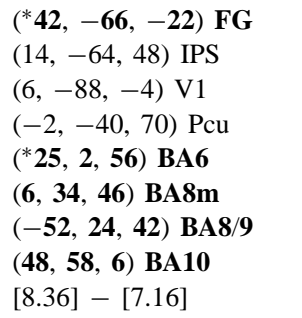 & $\begin{array}{l}(58,-28,54) \text { IPS } \\
(\mathbf{4 0}, 38,12) \text { BA9/46 } \\
(52,-38,2) \text { STS } \\
(-50,-48,42) \text { iPL } \\
(50,-66,42) \text { BA19 } \\
(-18,30,46) \text { BA6/8 } \\
{[6.03]-[5.60]}\end{array}$ & $\begin{array}{l}(\mathbf{*} \mathbf{1 2},-\mathbf{6 5}, \mathbf{5 6}) \mathbf{s P L} \\
(0,-54,60) \text { Pcu } \\
(42,-72,16) \text { BA39 } \\
(* 23,-78,33) \text { iIPS } \\
(\mathbf{2 5}, \mathbf{1}, \mathbf{5 5}) \text { BA6 } \\
(-\mathbf{4 6},-\mathbf{5 4},-\mathbf{6}) \text { V5 } \\
{[8.37]-[6.56]}\end{array}$ \\
\hline B.W. & $\begin{array}{l}(\mathbf{3 4},-\mathbf{5 2}, \mathbf{6 4}) \mathbf{s P L} \\
(32,-36,38) \mathrm{iPL} \\
\left({ }^{*} 13,-75,8\right) \mathrm{V} 1 \\
(\mathbf{4 8},-\mathbf{7 8},-\mathbf{6}) \text { iOG } \\
(* 38,-65,-15) \mathrm{FG} \\
(\mathbf{5 0}, \mathbf{4 0}, \mathbf{3 4}) \mathbf{B A 9} / \mathbf{4 6} \\
(\mathbf{3 2}, \mathbf{3 0}, \mathbf{0}) \mathbf{B A 4 7} \\
(\mathbf{3 2}, \mathbf{5 8},-\mathbf{4}) \mathbf{B A 1 0} \\
{[7.93]-[6.26]}\end{array}$ & $\begin{array}{l}\left({ }^{*} \mathbf{2 3},-\mathbf{5 6}, \mathbf{6 5}\right) \mathbf{s P L} \\
(48,-26,54) \text { iP/PoS } \\
(48,-76,-6) \text { iOG } \\
\left({ }^{*} 14,-68,16\right) \text { V1 } \\
(-54,-60,-2) \text { V5 } \\
(* 28,8, \mathbf{5 8}) \text { BA6 } \\
(\mathbf{4 8}, \mathbf{4 4}, \mathbf{3 4}) \text { BA9/46 } \\
{[8.45]-[7.52]}\end{array}$ & $\begin{array}{l}\left({ }^{*} 55,0,53\right) \text { CS } \\
(-42,-62,56) \text { sPL } \\
(30,-66,-4) \text { FG } \\
(50,38,16) \text { BA46 } \\
{[6.74]-[6.16]}\end{array}$ & $\begin{array}{l}(* 20,-60,61) \text { sPL } \\
(* 45,-9,56) \mathrm{iP} / \mathrm{PoS} \\
\left({ }^{*} 60,-55,-2\right) \mathrm{V} 5 \\
(* \mathbf{2 6}, \mathbf{1 1}, \mathbf{5 4}) \text { BA6 } \\
(\mathbf{4 6},-\mathbf{6 0}, \mathbf{2 6}) \text { BA39 } \\
(-\mathbf{2 6}, \mathbf{4 0},-\mathbf{1 2}) \text { BA11 } \\
{[8.58]-[6.89]}\end{array}$ \\
\hline S.T. & $\begin{array}{l}(\mathbf{3 4},-\mathbf{6 0},-\mathbf{2 2}) \mathbf{F G} \\
(38,-86,26) \text { BA1 } \\
\left({ }^{*},-98,-6\right) \text { V } 1 \\
(18,-62,-2) \text { LG } \\
(\mathbf{5 8}, \mathbf{1 6}, \mathbf{4 2}) \mathbf{B A 8} / \mathbf{i F S} \\
(-\mathbf{5 6}, \mathbf{2 6}, \mathbf{2 6}) \mathbf{B A 4 5} \\
\left({ }^{* 29,-57,52)} \mathbf{s P L}\right. \\
{[8.45]-[7.54]}\end{array}$ & $\begin{array}{l}(\mathbf{3 2},-\mathbf{7 0}, \mathbf{2 8}) \text { iIPS } \\
(* 2,-98,-6) \mathrm{V} 1 / \mathrm{LG} \\
(* 49,-26,41) \mathrm{iP} / \mathrm{PoS} \\
(* 34,-62,-22) \mathrm{FG} \\
(* 26,-60,60) \mathrm{sPL} \\
(* \mathbf{5 6}, \mathbf{2 2}, \mathbf{2 6}) \mathrm{BA45} \\
\left({ }^{* 32},-\mathbf{7}, \mathbf{5 3}\right) \mathbf{B A 6} \\
{[8.79]-[8.06]}\end{array}$ & $\begin{array}{l}(* 34,-27,71) \text { CS } \\
(\mathbf{0},-8,60) \text { BA6m } \\
(\mathbf{5 2},-\mathbf{4 6}, 2) \text { STS } \\
(-32,56,22) \text { BA10 } \\
(14,54,42) \text { BA9 } \\
(\mathbf{0},-6,44) \text { BA24 } \\
{[6.32]-[4.92]}\end{array}$ & $\begin{array}{l}\left({ }^{*} \mathbf{2 1},-\mathbf{7 5}, \mathbf{5 2}\right) \mathbf{s P L} \\
\left({ }^{*} 25,-72,38\right) \mathrm{iIPS} \\
\left({ }^{*} 49,-6,41\right) \mathrm{iP} / \mathrm{PoS} \\
\left({ }^{*} \mathbf{3 3}, \mathbf{3}, \mathbf{6 6}\right) \mathbf{B A 6} \\
\left({ }^{*} \mathbf{5 9},-\mathbf{6 5},-\mathbf{1}\right) \mathbf{V 5} \\
{[8.57]-[5.78]}\end{array}$ \\
\hline J.W. & 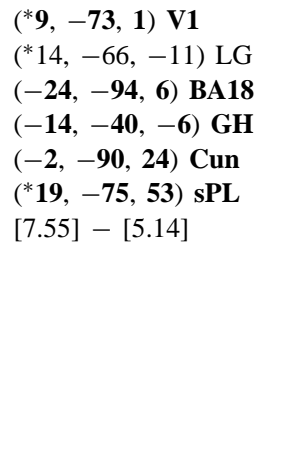 & 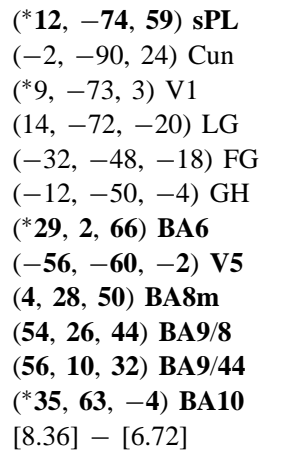 & $\begin{array}{l}(10,-98,2) \text { V1 } \\
(-62,8,24) \text { BA44 } \\
(16,-34,84) \text { BA5 } \\
(2,48,50) \text { BA8/9m } \\
{[7.27]-[6.40]}\end{array}$ & $\begin{array}{l}(* 9,-72,58) \text { sPL } \\
(41,-43,37) \text { iPL } \\
(-\mathbf{5 6},-62,-2) \text { V5 } \\
(\mathbf{0},-\mathbf{4 6}, \mathbf{1 0}) \text { BA30 } \\
(* 26,-3,56) \text { BA6 } \\
(\mathbf{3 6}, \mathbf{4 2},-\mathbf{1 4}) \text { BA47 } \\
(-\mathbf{4 6}, \mathbf{3 2}, \mathbf{4 4}) \text { BA8/9 } \\
(-\mathbf{5 0}, \mathbf{5 2}, 4) \text { BA10 } \\
(\mathbf{6}, \mathbf{2 6}, \mathbf{4 8}) \text { BA8m } \\
{[8.26]-[6.29]}\end{array}$ \\
\hline A.E. & $\begin{array}{l}(* 48,-78,1) \text { BA19/37 } \\
\left({ }^{* 28},-78,31\right) \text { iIPS } \\
(18,-80,-26) \text { LG } \\
(* 26,2,51) \text { BA6 } \\
(28,-88,18) \text { SOG } \\
\left({ }^{* 26,}-63,55\right) \text { sPL } \\
\left({ }^{* 34},-78,-23\right) \text { FG } \\
(-2,6,56) \text { BA6/8m } \\
(12,-32,78) \text { CS } \\
{[8.01]-[5.02]}\end{array}$ & $\begin{array}{l}(-\mathbf{4 4},-\mathbf{8 4}, \mathbf{4}) \mathrm{BA19/37} \\
(* 25,-62,62) \mathrm{sPL} \\
(30,-68,34) \mathrm{iIPS} \\
(* 35,-79,-24) \mathrm{FG} \\
(20,-82,-26) \mathrm{LG} \\
(* \mathbf{2 8},-\mathbf{5}, \mathbf{6 0}) \mathrm{BA6} \\
(60,14,26) \mathrm{BA} 6 / 9 \\
\left({ }^{* 52},-\mathbf{3 0}, \mathbf{4 3}\right) \mathrm{iP} / \mathrm{PoS} \\
(-\mathbf{2}, \mathbf{6}, \mathbf{5 8}) \mathbf{B A 6 m} \\
(\mathbf{0}, \mathbf{3 0}, \mathbf{4 6}) \mathbf{B A 8 m} \\
{[8.72]-[6.81]}\end{array}$ & $\begin{array}{l}(2,-36,74) \text { BA5m } \\
(-46,-72,40) \text { BA19/7 } \\
(* 51,-47,-29) \text { FG } \\
(-26,32,50) \text { SFS } \\
(68,0,-16) \text { BA21 } \\
(* 10,-47,-3) \text { GH } \\
{[7.91]-[6.25]}\end{array}$ & $\begin{array}{l}(* 25,-62,63) \text { sPL } \\
(* 52,-4,54) \text { iP/PoS } \\
\left({ }^{*} 29,-3,67\right) \text { BA6 } \\
(-\mathbf{3 4},-\mathbf{7 4}, \mathbf{3 0}) \text { BA39 } \\
(\mathbf{5 8},-\mathbf{5 4},-\mathbf{1 2}) \mathbf{V 5} \\
(\mathbf{4 0}, \mathbf{2}, \mathbf{3 2}) \mathbf{B A 6 i} \\
{[8.42]-[5.87]}\end{array}$ \\
\hline
\end{tabular}


Table 1 (Continued)

\begin{tabular}{|c|c|c|c|c|}
\hline S. & $\mathrm{I}-\mathrm{C}$ & $\mathrm{R}-\mathrm{C}$ & $\mathrm{I}-\mathrm{R}$ & R-I \\
\hline E.D. & $\begin{array}{l}(\mathbf{0},-86,-4) \text { V1 } \\
\left({ }^{* 12},-\mathbf{8 4}, \mathbf{4 6}\right) \text { IPS } \\
(-8,-72,60) \text { BA7 } \\
(\mathbf{4 2},-\mathbf{7 4},-\mathbf{1 4}) \text { BA19 } \\
(-\mathbf{3 4}, \mathbf{1 6}, \mathbf{6 2}) \text { BA6/8 } \\
{[7.75]-[6.29]}\end{array}$ & $\begin{array}{l}\left({ }^{*} \mathbf{1 6},-\mathbf{7 7}, \mathbf{5 3}\right) \mathbf{s P L} \\
(* 4,82,-1) \text { V1 } \\
\left({ }^{*} 8,-91,34\right) \text { iIPS } \\
(-32,-90,12) \text { BA } 19 \\
(-\mathbf{3 0}, \mathbf{1 0}, \mathbf{6 8}) \text { BA6 } \\
(* 43,-\mathbf{4 2}, \mathbf{5 5}) \mathbf{P o S} \\
(\mathbf{4 4},-\mathbf{7 0},-\mathbf{1 6}) \mathbf{B A 1 9 / 3 7} \\
{[7.95]-[6.73]}\end{array}$ & $\begin{array}{l}(2,-82,-10) \text { V1/LG } \\
(* 23,-71,-17) \text { FG } \\
(-68,-36,0) \text { BA21 } \\
(-10,-42,-6) \mathbf{G H} \\
(34,-20,70) \mathbf{C S} \\
{[7.05]-[6.52]}\end{array}$ & $\begin{array}{l}(* 31,-69,25) \text { iIPS } \\
(8,-66,58) \text { sPL } \\
(32,-8,54) \text { PrS } \\
(36, \mathbf{1 0}, \mathbf{5 8}) \text { sFS } \\
{[7.03]-[4.93]}\end{array}$ \\
\hline
\end{tabular}

${ }^{a}$ Only the most significant voxel of each differentiated focus is given. The non-bold coordinates are the most significant voxels from relevant sub-foci within. All foci, as sub-foci within a larger region, are reported in order of decreasing significance level. In case of bilateral activation, only the mean of the two absolute coordinates is reported (indicated by ' $*$ ' in superscript). Also, at the bottom of each column, Z-values of respectively, the first and the last element are reported. Abbreviations: BA, Brodmann area; CS, central sulcus; Cun, cuneus; FG, fusiform gyrus; FS, frontal sulcus; GH, parahippocampal gyrus; iP, inferieur parietal; IPS, intraparietal sulcus; LG, lingual gyrus; OG, occipital gyrus; PL, parietal lobe; PoS, post-central sulcus; PrS, pre-central sulcus (s, superior; i, inferior; m, medial).

Institute (MNI) template, smoothing and construction of statistical parametric maps. For each individual subject, the two $\mathrm{CI}$ and the two $\mathrm{CR}$ time series were analyzed separately. The four remaining time series (IR and RI) were analyzed together. Regions were selected for activation height and spatial extent and were considered to be activated significantly if the resulting corrected $P$-value was $<0.05$. This analysis was performed using voxels of $2 \mathrm{~mm} \times 2 \mathrm{~mm} \times 2 \mathrm{~mm}$. The corresponding group analysis (on the pooled data of all subjects) was performed with a lower resolution $(3 \mathrm{~mm} \times 3 \mathrm{~mm} \times 3 \mathrm{~mm})$.

\section{RESULTS}

\subsection{Behavioral data}

\subsubsection{Reaction times (RTs)}

Overall, mean reaction time was $1750 \mathrm{~ms}$. The analysis revealed a significant main effect of process, $F(1,5)=84.47$, $P<0.001$, indicating that, generally, the mental rotation trials were executed more slowly than the invariance trials (means were 2297 and $1473 \mathrm{~ms}$, respectively). Also, a significant main effect of the angular difference (AD) was found, $F(11,55)=3.79, P<0.001$.

The two-way interactions process $\times \mathrm{AD}$ and match $\times \mathrm{AD}$ both turned out significantly (respectively, $F(11,55)=$ 5.78, $P<0.001$ and $F(11,55)=8.98, P<0.001)$. The process $\times \mathrm{AD}$ interaction points to a differentiating effect of the angular difference in the two experimental conditions. A Tukey (HSD) post-hoc analysis revealed no differences between RTs in the invariance condition, while these differences were obtained in the rotation condition. A similar observation can be made regarding the match $\times$ AD-interaction: differences between reaction times for match trials are present, but absent for non-match trials.

The three-way interaction process $\times$ match $\times \mathrm{AD}$ was found to be highly significant, $F(11,55)=11.76, P<$ 0.001, and can be seen in Fig. 2. Trials in the rotation con- dition showed a clear dependency on the angular difference between the objects. A linear regression on mean RTs on match trials per value of $\mathrm{AD}$ estimated the speed of rotation to be $186^{\circ} / \mathrm{s}$.

\subsubsection{Accuracy}

The overall mean proportion of correct responses was 0.79 . No significant main effects were observed. For the two-way interactions, only the match $\times$ AD-effect reached a significant level, $F(11,55)=3.59, P<0.001$ (the interaction process $\times \mathrm{AD}$ did not, $F(11,55)<1)$. A Tukey (HSD) post-hoc analysis indicated that only for large values of $\operatorname{AD}\left(120,150\right.$ and $\left.180^{\circ}\right)$ accuracy rates on the match and non-match trials differed significantly.

The interaction between the three variables turned out significantly, $F(11,55)=2.38, P=0.02$ and is depicted in Fig. 3.

\subsection{Imaging data}

Table 1 summarizes the actual Talairach coordinates [41] of both the group analysis and the individual analyses. We limit the discussion to the results of the group study.

Invariance-control (I-C, see Fig. 4a): We have found a large, differentially activated region that covers most of the occipital lobe, both medial and lateral, striate (V1) and extrastriate (BA18 and 19) cortex. Strongest activations were registered in the two primary projection areas. In the so-called "ventral stream", which originates from these areas, the most significant foci were the right inferior occipital gyrus (BA18/19) and the fusiform gyrus (BA19/37), bilaterally. Also in both hemispheres, the superior parietal cortex (BA7) was identified, while this activation seems to be concentrated in the intraparietal sulcus (IPS). Finally, two smaller frontal areas can be seen: one in the right pre-central sulcus (BA6), location of the frontal eye fields (FEF; [35]), and one in the medial part of BA8.

Rotation-control (R-C, see Fig. 4b): All areas found in the previous contrast were also visible in this contrast. The 
(a)

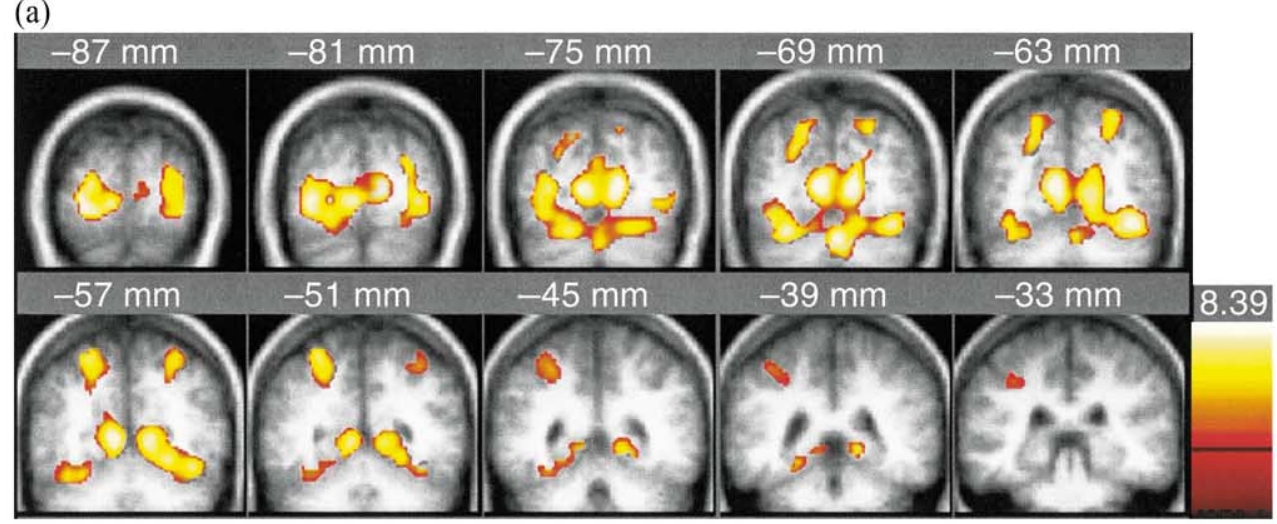

(b)

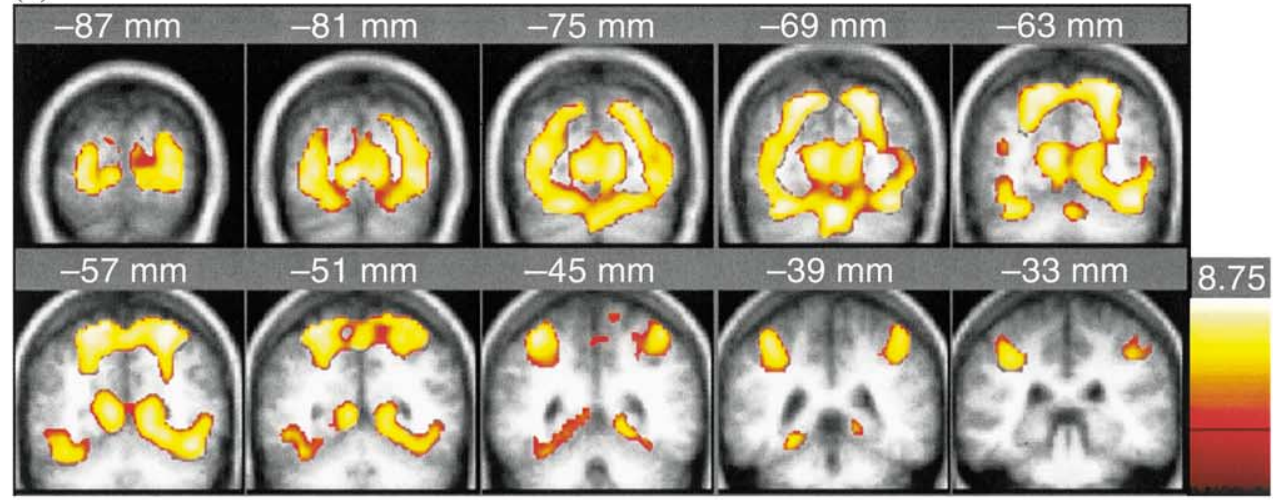

(c)

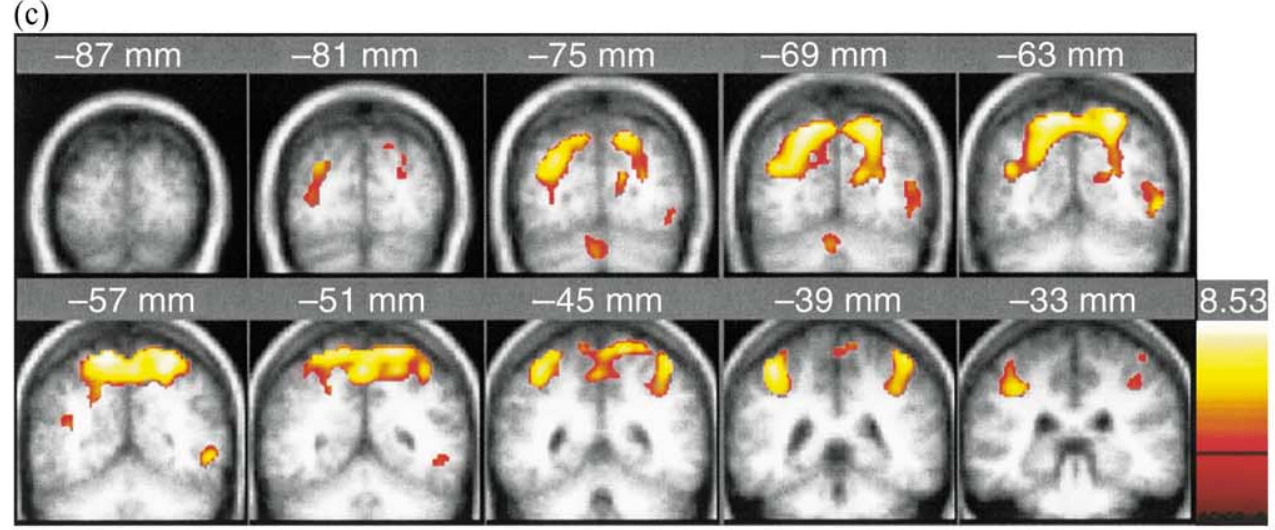

Fig. 4. Activation foci of the group analysis, plotted on 10 coronal slices of the mean normalized anatomy of all subjects (posterior ( -87 ) to anterior $(-33)$, each $6 \mathrm{~mm}$ ), for the three contrasts: (a) invariance-control; (b) rotation-control; and (c) rotation-invariance. The color code indicates maximal $Z$-value for each contrast.

three large clusters (occipital and BA7, bilateral) seem to form one network, which covers the dorsal as well as the ventral stream. The strongest signal was now observed in the two superior parietal lobes (BA7). Moreover, this activation seems to spread out, via the lateral part of the IPS, to the inferior parietal region (BA40) and the post-central sulcus.

In the ventral stream, activation in BA19/37 (the posterior fusiform gyrus) was found, in the two primary visual areas, in the cuneus and in additional occipital areas (BA18/19), all bilaterally. The activation in the anterior lingual gyrus was mainly located in the left hemisphere.

Frontally, BA6 could be identified bilaterally. Both foci included the pre-central sulcus $(\operatorname{PrS})$ as well as the superior frontal sulcus (SFS). ${ }^{4}$ In addition, the right cluster demonstrated higher signal intensity in a dorsolateral area that lay

\footnotetext{
${ }^{4}$ Following the $y$-axis, activation was found from -9 to $+15 /+18$ for the left and the right area, respectively.
} 
(a)

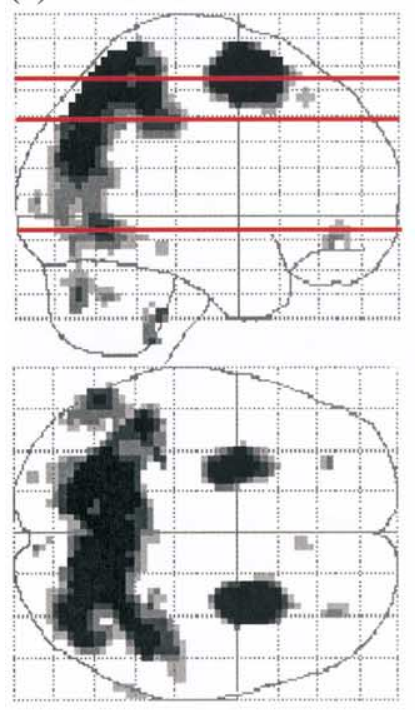

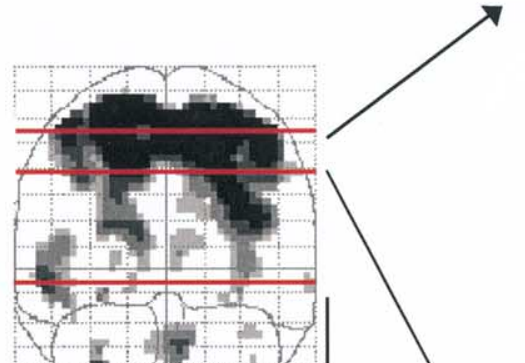

(b)

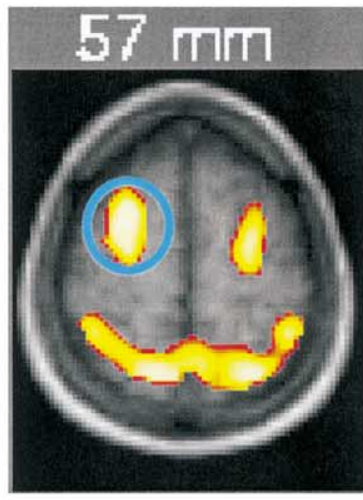

(c)

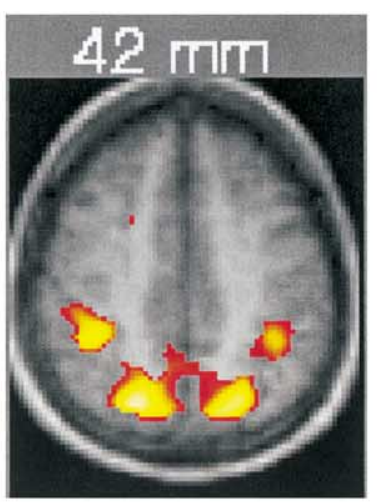

8.53 (d)

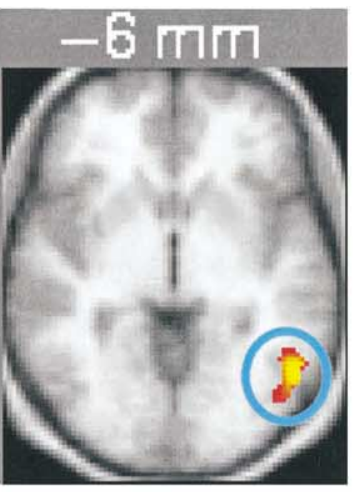

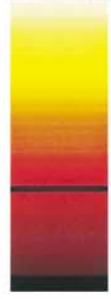
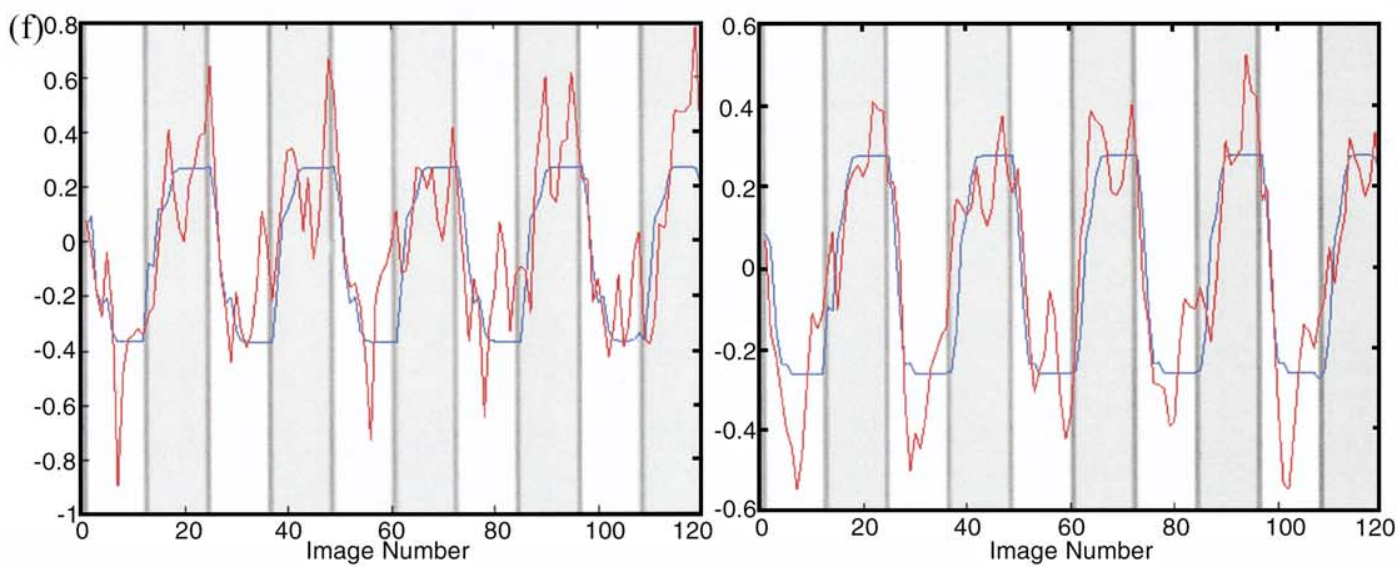

Fig. 5. (a) SPM of the R-I contrast on a saggital, coronal and transversal view of a "glass brain". Three transversal slices were selected (red lines) and plotted on the mean normalized anatomy of all subjects; (b) $Z=57 \mathrm{~mm}$, the superior parietal lobes (BA7) and BA6 (PrS and SFS), bilateral; (c) $Z=42 \mathrm{~mm}$, the intraparietal sulcus (to the post-central sulcus), bilateral; (d) $Z=-6 \mathrm{~mm}$, V5/MT, left, unilateral; (e) color code depicting maximal $Z$-value for the three slices; and (f) time course of the averaged MR signal of the most significant voxel in the area in the blue circle: V5/MT (left plot) and right BA6 (right plot). The signal (red curve) is plotted as a function of image number. The blue curve depicts the modeled signal. Dark hatching: indication image; light hatching: rotation condition; no hatching: invariance condition. 
between BA6 and BA9/46. Finally, the medial part of BA8 was identified.

Rotation-invariance (R-I, see Figs. 4c and 5): A somewhat similar pattern as in the $\mathrm{R}-\mathrm{C}$-contrast was observed. However, none of the regions in the ventral stream (i.e. the occipito-temporal areas), neither BA9/46 nor the medial part of BA8 were differentially activated in this contrast. Posterior, we found strong activations in the two superior parietal lobes. These bilateral activations seem to spread to the inferior parietal cortex and post-central sulcus, via the lateral part of the IPS. The activation in the inferior part of the IPS (BA7/19/18), however, is primarily present in the right hemisphere. Again, the pre-central and superior frontal sulci can be clearly observed.

In the left lateral-temporal area, on the border of the middle and inferior temporal gyri (BA19/37), a smaller focus of activation was present. This location resembles V5/MT [56], an area, which was also reported in a mental rotation study by Cohen et al. [7].

Invariance-rotation (I-R): Compared to the I-C-contrast, a totally new pattern emerged. Among the differentially activated regions were the left BA21, a large region located around the medial part of BA6, the dorsal surface of BA44 in the right hemisphere, and the left central sulcus and BA22. However, these activations turned out to be deactivations relative to the indication conditions with larger deactivations during rotation than during invariance. Because the significance of these values was caused by a negative value of the rotation component, the I-R-contrast did not yield interpretable results.

\section{Discussion}

\subsection{Behavioral results}

The process $\times \mathrm{AD}$ interaction for reaction times and the three-way interactions of both behavioral measures all indicate a differentiating effect of the specific viewpoint at which the objects were presented. The effect of AD in the rotation condition clearly points to a viewpoint-dependent component. The classical finding of an increasing reaction time with increasing value of $\mathrm{AD}$ for match trials is consistent with an explanation in terms of an analogue mental rotation process. ${ }^{5}$ The deterioration of performance in terms of accuracy confirms this point. In contrast, changes

\footnotetext{
${ }^{5}$ In Figs. 2 and 3, one can observe a differential effect of AD for non-match compared to match trials: there is a trend towards shorter response times and higher accuracy for larger angular differences. The explanation for this discrepancy can be found in the way the mirrored objects were constructed and the specific "anchor" subjects use to mentally rotate an object (i.e. which components have to be aligned in order to compare the two objects). If a subjects uses the two co-planar side-components as anchor, a theoretically "large" $\mathrm{AD}$ in fact constitutes a "small" $\mathrm{AD}$ for the subject and vice versa. For a more detailed discussion of this issue, see [48].
}

in viewpoint did not significantly affect behavioral measures when recognition was to be based on invariant features. Note hereby that one single projected angle could not have been regarded as invariant. Indeed, as Willems and Wagemans [57] showed, the presented viewpoint of a projected non-orthogonal angle, embedded in a solid cross-like figure, systematically influenced subject's perception of that angle. However, in the present experiment, all three angles were altered, allowing for a veridical interpretation of the angles over all viewpoints.

As expected, the analysis of the data on the behavioral level confirmed the fundamental distinction between the recognition processes in the two experimental conditions. Now, we turn to the neurofunctional results.

\subsection{Neurofunctional results}

Inspection of Fig. 4a and b already confirms the dissociation-hypothesis: not all significantly activated regions are present in both conditions. Although all the significantly activated regions in the invariance condition were also activated in the rotation condition, some areas are uniquely activated by the latter. The dissociation-hypothesis receives further support by the presence of significant areas in the R-I-contrast. Moreover, compared to the control condition, a dissociation is also apparent in the location of the most significant area. As predicted, the main focus of activation was in occipito-temporal areas in the invariance condition, and in the parietal region in the rotation condition.

\subsubsection{Common activations}

Most relevant in this investigation is the ventral, occipito-temporal stream, present in both conditions. Most significant in this stream was the bilateral focus in the fusiform gyrus. Locations in these regions have been repeatedly reported in form, shape or object recognition studies (e.g. $[1,2,6,23,29])$. However, the precise location of the activity is rather variable and presumably strongly influenced by the specific stimuli and task. The fact that these regions have also been associated with shape processing of nonsense stimuli (e.g. [23,33]), i.e. irrespective of meaning or structural representations stored in memory, is compatible with the task and the objects used in this study. If it is the case that activation in these areas is related to bottom-up construction of shape description [23], one would indeed expect these areas to be activated in both conditions.

An intriguing observation is the asymmetry in the foci in the inferior occipital gyrus in the invariance condition. Although these areas are also apparent in the rotation condition, it is only in the invariance condition that the activity is clearly more pronounced in the right hemisphere. It is more extended (see Fig. 4a, from -87 to -69 ), but also more significant (maximal $Z$-value on the right was 8.27, on the left 7.23). Moreover, this area is located in the dorsal stream, but more medial and more posterior than LOC (lateral-occipital complex, [29]), which is supposed to code parts of an object. 
One possibility would then be that the extraction of more elementary features (such as angles) is associated with this region. The fact that the invariance condition requires a relatively precise discrimination could shed some light on the right-hemispheric dominance: there is evidence that subordinate object recognition or the visual processing of specific visual forms is primarily a function of the right hemisphere [25,30,31].

\subsubsection{Rotation-unique activation}

The areas that will be discussed next are either uniquely (SFS, inferior part of IPS, V5/MT) or clearly more strongly (BA7/IPS, FEF) activated by the rotation condition, compared to the invariance condition. Unless explicitly mentioned, the areas are significant in both contrasts $(\mathrm{R}-\mathrm{C}$ and $\mathrm{R}-\mathrm{I})$.

First, there is the activity in the inferior part of the IPS, the superior parietal lobes and the lateral part of the IPS. The importance of the posterior parietal cortex in spatial processes in general and the mental rotation process in particular is unquestionable, but recent studies have also specifically emphasized the functional role of the IPS. Faillenot et al. [14] claim this area to be involved in the integration of 3-D features of objects, while Goebel et al. [17] relate the posterior T-junction of the IPS to spatial transformation. Although the activation spreads out more anteriorly, we have also identified the lateral part of IPS in the R-I-contrast, which seems to confirm the latter hypothesis.

A striking result in the rotation condition concerns the presence of two extensive clusters in BA6 (see Fig. 5). These included, amongst others, the FEF at the border of the superior frontal (SFS) and pre-central sulcus (PrS), a focus that was also apparent in the I-C-contrast, but to a smaller extent. Although voluntary eye-movements are surely a plausible and likely candidate to explain this result, we suspect that this might not be the only explanation. ${ }^{6}$ Indeed, in the Carpenter et al. [5] study, these areas were also more strongly activated than in a grid-scanning condition involving a large number of eye-movements. We, therefore, agree that this might be related to the greater computational demand in computing the object's spatial presentation, since

\footnotetext{
${ }^{6}$ Just and Carpenter [22] analyzed eye-movements during the execution of a mental rotation task. They concluded that subjects rotated individual components, which implies that all corresponding components in both figures had to be re-fixated over and over. Obviously, this process is not necessary in the invariance condition. Therefore, it seems plausible to state that subjects made more eye-movements while performing a trial in the rotation condition. However, they performed more trials in the invariance condition. This way, it could be that the total number of eye-movements (between the two objects) during one epoch was more or less equal. To investigate this, we monitored the movements of the right eye of one subject (E.D.), by using an MR-compatible infrared eye-movement registration device (Ober2, Permobil MeditechAB, Sweden). For the RI and IR time series, we counted the number of horizontal saccades per experimental block. A $t$-test confirmed that the number of saccades did not differ significantly for the two experimental conditions, $t(19)=1.83$, $P>0.05$
}

this is likely to be a more demanding process in the rotation condition.

The extensive areas located in BA6 also comprise the SFS. An area in this sulcus has been related to spatial working memory [9]. It is obvious that spatial memory plays a more significant part in the rotation condition. In the invariance condition, the only spatial information that has to be remembered once the nature of the object (i.e. orthogonal or skewed) has been determined, is of which object the information was collected. In the rotation condition, a constant update is needed (e.g. which component was rotated). Clearly, this activation has to be considered in relation to the activation in the previously mentioned posterior parietal areas.

Finally, we give additional evidence for the co-activation of area V5/MT. Although no actual motion was present, we still observed activation in the area known as the "motion"-area. However, to what extent this activation is a consequence of the "perception" of imaginary motion or a necessary component in the execution of that imagery motion, is not known. Interestingly, in the R-I-contrast, the area can only be seen in the left hemisphere (see Fig. 5d). Nevertheless, looking at individual results, this was only the case in two subjects. Other subjects showed bilateral activation (2), or merely on the right (1), or did not have visible activation in the area at all (1).

Our findings on the mental rotation process, are thus, in (close) agreement with those of previous studies. The three large-scale neural systems reported by Carpenter et al. [5], parietal, occipito-temporal, and frontal, were visible compared to the baseline. The direct comparison with the invariance condition confirmed the importance of the parietal cortex in mental rotation, as well as confirming that occipito-temporal activation is not specifically related to this process. Finally, we failed to find any convincing evidence for the hemispheric lateralization of mental rotation (see also [5]).

Although the reason for including the rotation condition in the present investigation was not primarily to focus on the mental rotation process per se, our findings on mental rotation nevertheless, provide some contribution to the mental rotation literature. As a baseline for comparing cortical activations in the mental rotation condition, previous studies have used either the same objects presented with an angular difference of $0^{\circ}$ [7] or a different task [5,39]. However, as yet there has been no direct comparison between a mental rotation condition and an equivalent task employing a single paradigm and similar objects across conditions. The present study does provide such a comparison, and thus, confirms the validity of previously reported findings.

\section{General conclusions}

We developed two conditions in which we used a highly similar stimulus set and an identical paradigm, namely 
a same-different judgment task. The crucial variable in both conditions was the angular difference between the two presented views. Our results are compatible with the existence of two different "routes" involved in object perception, which are dissociable on both the behavioral and the neurofunctional level.

The dorsal-ventral dissociation apparent in the neurofunctional data closely resembles the functional dissociation in the model of Milner and Goodale [32]. Indeed, in their account, it is postulated that both the dorsal and the ventral stream process information about object features, but that this information serves different purposes. If the goal of the visual analysis is to (consciously) recognize or categorize an object, the ventral stream encodes the information in an environmental reference system (viewpoint-independent). The dorsal stream, on the other hand, is concerned with the programming and visual control of actions, and transforms the information in egocentric (viewpoint-dependent) coordinates. In so far as mental rotation is an action performed on an object, our results clearly fit with this hypothesis.

The lack of invariance-specific activations leaves us with a single dissociation instead of the double dissociation, which would otherwise have been stronger evidence for the existence of "multiple routes" in the brain. Hence, one could still argue that all the activity is in fact situated in a single neural system and that the supplementary regions activated in the rotation condition simply indicate that the task in this condition was relatively difficult, while the regions activated in common could reflect lower-level recognition processes essential to both tasks. In other words, the supplementary activated regions in the rotation condition might be a mere manifestation of increased difficulty within a single system, not a separate, alternative route. Indeed, such an interpretation of the result does seem more parsimonious at first sight and we surely do not rule out the possibility that some part of the activations observed in the rotation condition might be due to increased difficulty or attentional demands (e.g. [59]).

However, it is unlikely that this is the sole source of activation in these areas: the subtractions rotation minus invariance and rotation minus control yield quite similar results (even when taking the range of $Z$-values into account), despite the fact that the invariance condition is clearly much more demanding than the control condition. In addition, it is quite difficult to see how such an account can then be reconciled with the neuropsychological evidence mentioned in the Section 1. Moreover, a recent study by Warrington and Davidoff [55] described a patient, J.A.B., whose performance on rotational tasks was determined by her inability to identify the objects. That is, she could execute mirror discriminations almost exclusively for objects she could not identify. Distinguishing between mirror images of objects she could identify was gravely impaired. Warrington and Davidoff argue that this represents an inhibition between two major streams of information in the visual system: when there is no access to an object-centered representation, processing proceeds to "the limits of the observer's spatial abilities" ([55], p. 1232). However, when identification was achieved by means of an object-centered representation, there is a disconnection from the required allocentric object spatial position coordinates. In any case, such a pattern of results would be highly unlikely if the observations found in the present study were the result of processing within a single system. For these reasons, we are reluctant to withhold this alternative, but favor the "different routes" interpretation, which can handle those findings in a more straightforward manner.

To conclude, if we assume the initial construction of a stimulus representation to be a purely stimulus-driven perceptual process, located in the fusiform gyri [23], there should be noticeable activation in this region in both the rotation and the invariance conditions. This was indeed the case. However, because this "route" does not suffice to perform the rotation condition, additional areas in the dorsal stream are activated in which the construction of explicitly viewer-centered stimulus representations takes place (cf. [45]).

\section{Acknowledgements}

This research was supported by grants to J.W. from the Research Program of the Fund for Scientific ResearchFlanders, Belgium (FWO G.0130.98) and from the Research Council at the University of Leuven (IDO/98/002). We would like to thank Toon Sykora for programming the behavioral paradigm and Moshe Bar and an anonymous reviewer for their helpful comments on the previous version of this article.

\section{References}

[1] Bar M, Tootell R, Schacter D, Greve D, Fischl B, Mendola J, et al. Cortical mechanisms specific to explicit visual object recognition. Neuron 2001;29:529-35.

[2] Beason-Held LL, Purpura KP, Van Meter JW, Azari NP, Mangot DJ, Optican LM, et al. PET reveals occipito-temporal pathway activation during elementary form perception in humans. Visual Neuroscience 1998;15:503-10.

[3] Biederman I, Bar M. One-shot viewpoint invariance in matching novel objects. Vision Research 1999;39:2885-99.

[4] Biederman I, Gerhardstein PC. Recognizing depth-rotated objects: evidence and conditions for three-dimensional viewpoint invariance. Journal of Experimental Psychology: Human Perception \& Performance 1993;19:1162-82.

[5] Carpenter PA, Just MA, Keller TA, Eddy W, Thulborn K. Graded functional activation in the visuo-spatial system with the amount of task demand. Journal of Cognitive Neuroscience 1999;11:9-24.

[6] Clark VP, Maisog JM, Haxby JV. fMRI study of face perception and memory using random stimulus sequences. Journal of Neurophysiology 1998;79:3257-65.

[7] Cohen MS, Kosslyn SM, Breiter HC, DiGirolamo GJ, Thompson WL, Anderson AK, et al. Changes in cortical activity during mental rotation: a mapping study using functional MRI. Brain 1996;119:89-100. 
[8] Cooper ACG, Humphreys GW. Task-specific effects of orientation information: neuropsychological evidence. Neuropsychologia 2000;38:1607-15.

[9] Courtney SM, Petit L, Maisog JM, Ungerleider LG, Haxby JV. An area specialized for spatial working memory in human frontal cortex. Science 1998;279:1347-51.

[10] Courtney SM, Ungerleider LG. What fMRI has taught us about human vision. Current Opinion in Neurobiology 1997;7:554-61.

[11] Davidoff J, Warrington EK. The bare bones of object recognition: implications from a case of object recognition impairment Neuropsychologia 2000;37:279-92.

[12] Edelman S. Class similarity and viewpoint invariance in the recognition of 3-D objects. Biological Cybernetics 1995;72:207-20.

[13] Eley MG. Identifying rotated letter-like symbols. Memory \& Cognition 1982;10:25-32

[14] Faillenot I, Toni I, Decety J, Grégoire M-C, Jeannerod M. Visual pathways for object-oriented action and object recognition: functional anatomy with PET. Cerebral Cortex 1997;7:77-85.

[15] Farah MJ, Aguirre GK. Imaging visual recognition: PET and fMRI studies of the functional anatomy of human visual recognition. Trends in Cognitive Sciences 1999;3:179-86.

[16] Farah MJ, Hammond KM. Mental rotation and orientation-invariant object recognition: dissociable processes. Cognition 1988;29:29-46.

[17] Goebel R, Linden DEJ, Lanferman H, Zanella F, Singer W. Functional imaging of mirror and inverse reading reveals separate co-activated networks for oculomotion and spatial transformation. Neuroreport 1998;9:713-9.

[18] Grill-Spector K, Kushnir T, Edelman S, Avidan G, Itzchak Y, Malach R. Differential processing of objects under various viewing conditions in the human lateral occipital complex. Neuron 1999;24:187-203.

[19] Hayward WG, Tarr MJ. Testing conditions for viewpoint invariance in shape recognition. Journal of Experimental Psychology: Human Perception \& Performance 1997;23:1511-21.

[20] Hayward WG, Williams P. Viewpoint dependence and object discriminability. Psychological Science 2000;11:7-12.

[21] Humphreys GW, Riddoch MJ. Routes to object constancy: implications from neurological impairments of object constancy. Quarterly Journal of Experimental Psychology 1984;37A:469-95.

[22] Just MA, Carpenter PA. Cognitive coordinate systems: accounts of mental rotation and individual differences in spatial ability. Psychological Review 1985;92:137-72.

[23] Kanwisher N, Woods RP, Iacoboni M, Mazziotta JC. A locus in human extrastriate cortex for visual shape analysis. Journal of Cognitive Neuroscience 1997;9:133-42.

[24] Karnath H-O, Ferber S, Bülthoff HH. Neuronal representation of object orientation. Neuropsychologia 2000;38:1235-41.

[25] Koutstaal W, Wagner AD, Rotte M, Maril A, Buckner RL, Schacter DL. Perceptual specificity in visual object priming: functional magnetic resonance imaging evidence for a laterality difference in fusiform cortex. Neuropsychologia 2001;39:184-99.

[26] Kosslyn SM, Alpert NM, Thompson WL, Chabris CF, Rauch SL, Anderson AK. Identifying objects seen from different viewpoints: A PET investigation. Brain 1994;117:1055-71.

[27] Kraut M, Hart J, Soher BJ, Gordon B. Object shape processing in the visual system evaluated using functional MRI. Neurology 1997:48:1416-20.

[28] Lawson R. Achieving visual object constancy across plane rotation and depth rotation. Acta Psychologica 1999;102:221-45.

[29] Malach R, Reppas JB, Benson RR, Kwong KK, Jiang H, Kennedy WA, et al. Object-related activity revealed by functional magnetic resonance imaging in human occipital cortex. In: Proceedings of the National Academy of Sciences of the USA 1995;92:8135-39.

[30] Marsolek CJ. Abstract visual-form representations in the left cerebral hemisphere. Journal of Experimental Psychology: Human Perception \& Performance 1995;21:375-86.

[31] Marsolek CJ. Dissociable neural subsystems underlie abstract and specific object recognition. Psychological Science 1999;10:111-8.
[32] Milner AD, Goodale MA. The visual brain in action. Oxford: Oxford University Press, 1995.

[33] Op de Beeck H, Béatse E, Wagemans J, Sunaert S, Van Hecke P. The representation of shape in the context of visual object categorization tasks. Neuroimage 2000;12:28-40.

[34] Op de Beeck H, Vogels R. Spatial sensitivity of macaque inferior temporal neurons. Journal of Comparative Neurology 2000;426: $505-18$.

[35] Paus T. Location and function of the human frontal eye-field: a selective review. Neuropsychologia 1996;34:475-83.

[36] Richter W, Somorjai R, Summers R, Jarmasz M. Motor area activity during mental rotation studied by time-resolved single-trial fMRI. Journal of Cognitive Neuroscience 2000;12:310-20.

[37] Shepard RN, Metzler J. Mental rotation of three-dimensional objects. Science 1971;171:701-3.

[38] Sugio T, Nakai T, Miki Y, Togashi K, Konishi J. The neural basis of viewpoint compensation in human object recognition: an fMRI study. In: Proceedings of the Poster presented at the 6th Annual Meeting of the Organization for Human Brain Mapping, San Antonio, Texas, USA, 2000

[39] Tagaris GA, Kim S, Strupp JP, Andersen P, Ugurbil K, Georgopoulos AP. Mental rotation studied by functional magnetic resonance imaging at high field $(4 \mathrm{~T})$ : performance and cortical activation. Journal of Cognitive Neuroscience 1997;9:419-32.

[40] Takano Y. Perception of rotated forms: a theory of information types. Cognitive Psychology 1989;21:1-59.

[41] Talairach J, Tournoux P. Co-planar stereotaxic atlas of the human brain. New York: Thieme, 1988

[42] Tarr MJ. Rotating objects to recognize them: a case study on the role of viewpoint-dependency in the recognition of three-dimensional objects. Psychonomic Bulletin \& Review 1995;2:55-82.

[43] Tarr MJ, Bülthoff HH. Is human object recognition better described by geon structural descriptions or by multiple views? Comment on Biederman and Gerhardstein in 1993. Journal of Experimental Psychology: Human Perception \& Performance 1995;21:1494-505.

[44] Tootell RBH, Hadjikhani NK, Mendola JD, Marrett S, Dale AM. From retinotopy to recognition: fMRI in human visual cortex. Trends in Cognitive Sciences 1998;2:174-83.

[45] Turnbull OH, Carey DP, McCarthy RA. The neuropsychology of object constancy. Journal of the International Neuropsychological Society 1997;3:288-98.

[46] Turnbull OH, McCarthy RA. When is a view unusual? A single case study of orientation-dependent visual agnosia. Brain Research Bulletin 1996;40:497-503.

[47] Van Lier R, Wagemans J. From images to objects: global and local completions of self-occluded parts. Journal of Experimental Psychology: Human Perception \& Performance 1999;25:1721-41.

[48] Vanrie J, Willems B, Wagemans J. Multiple routes to object matching from different viewpoints: mental rotation versus invariant features. Perception 2001;30 (in press).

[49] Vecera SP. Visual object representation: an introduction. Psychobiology 1998;26:281-308.

[50] Vogels R, Biederman I, Bar M, Lorincz A. Inferior temporal neurons show greater sensitivity to non-accidental than to metric shape differences. Journal of Cognitive Neuroscience 2001;13:444-53.

[51] Vogels R, Orban GA. Coding of stimulus invariances by inferior temporal neurons. In: Norita M, Bando T, Stein B, editors. Progress in Brain Research 1996;112:195-211.

[52] Wagemans J, Van Gool L, Lamote C. The visual system's measurement of invariants need not itself be invariant. Psychological Science 1996;7:232-6.

[53] Wagemans J, Van Gool L, Lamote C, Foster DH. Minimal information to determine affine shape equivalence. Journal of Experimental Psychology: Human Perception \& Performance 2000;26:443-68.

[54] Wagemans J, Verstraten FAJ, He S, editors. Beyond the decade of the brain: towards a functional neuroanatomy of the human mind. Acta Psychologica (special issue) 2001;107(1-3):1-354. 
[55] Warrington EK, Davidoff J. Failure at object identification improves mirror image matching. Neuropsychologia 2000;38: 1229-34.

[56] Watson JDG, Myers R, Frackowiak RSJ, Hajnal JV, Woods RP, Mazziotta JC, et al. Area V5 of the human brain: evidence from a combined study using positron emission tomography and magnetic resonance imaging. Cerebral Cortex 1993;3:79-94.

[57] Willems B, Wagemans J. The viewpoint-dependency of veridicality: psychophysics and modeling. Vision Research 2000;40: 3017-27.

[58] Willems B, Wagemans J. Matching multi-component objects from different viewpoints: mental rotation as normalization? Journal of Experimental Psychology: Human Perception \& Performance 2001;27 (in press).

[59] Wojciulik E, Kanwisher N. The generality of parietal involvement in visual attention. Neuron 1999;23:747-64. 